\title{
Multi-scale foam behaviour characterisation
}

\author{
P. Viot ${ }^{1} \&$ D. Bernard ${ }^{2}$ \\ ${ }^{1}$ LAMEFIP, ENSAM de Bordeaux, Talence Cedex, France \\ ${ }^{2} I C M C B, C N R S$, Université Bordeauxl, PESSAC, France
}

\begin{abstract}
The mechanical behaviour of polymeric foams depends on several parameters such as temperature, material density and strain rate. The identification of the parameters characterizing this behaviour under dynamic loading requires the design of special apparatus like a fly wheel, drop tower or Hopkinson bars, allowing high compression speeds.

The foams studied here are multi-scale materials; the agglomerated beads (mesoscopic scale, millimetric diameters) are composed of microscopic closed cells (a few tens of microns). Constitutive materials of these foams are polypropylene, polystyrene and cork. The response of the material to a dynamic loading consists of three regions: an elastic phase, a plastic phase and densification. The first part of this work deals with the identification of the behaviour of these multi-scale foams as a function of density and strain rate. In the second part, original observations of the physical phenomena initiated during the yield plateau are presented and analysed. Buckling of bead and cell wall and strong localisation of damage were studied using several devices and techniques such as high speed cameras, SEM, and micro tomography.

Keywords: multi-scale foam, cellular material, dynamic loading.
\end{abstract}

\section{Introduction}

Polymer foams are used in many applications of passive safety for consumer goods (packaging for electronic equipment...) or for consumers themselves (helmets, knee pads...). It is usual to classify cellular materials in closed or opened cell foams [1], but it is also important to distinguish between foams which are constituted only of micro cells (commonly used in large plates for thermal or sound isolation applications in civil engineering) and those composed of fused beads (their size is millimetric and constitute the mesostructure) which are themselves made of micro cells (figures 1 and 2). 

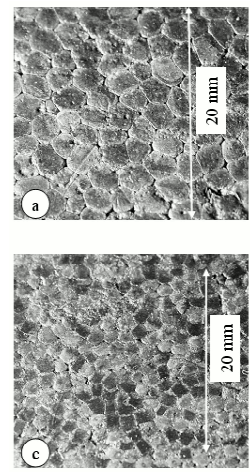
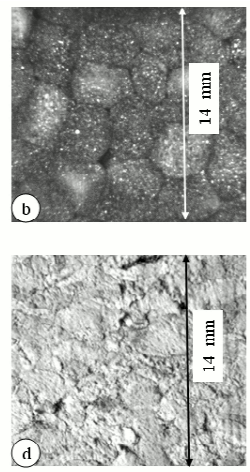

Figure 1: Pictures of multi-scale foams (a) polypropylene foam (knauf), polypropylene foam (JSP), (c) polystyrene foam (knauf), (d) cork.
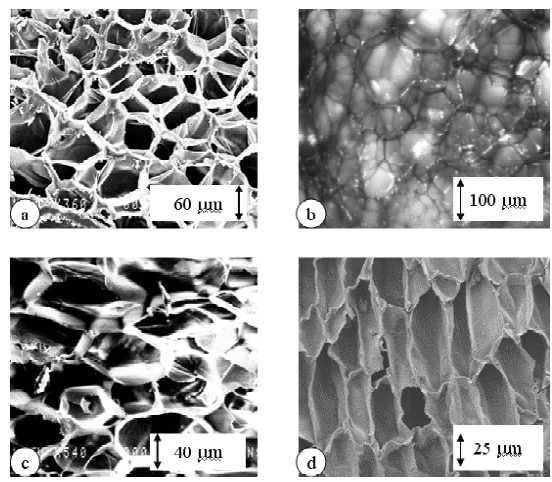

Figure 2: $\quad$ SEM of multi-scale foams (a) polypropylene foam (knauf), (b) polypropylene foam (JSP), polystyrene foam (knauf), (d) cork.

These last foams are multi-scale materials. The mesoscopic morphology of these foams is determined during the manufacturing process: expanded plastic foam beads are injected into a mould where individual beads are fused together under steam heat and pressure. The constitutive material of these foams can be a polymer such as polypropylene (figures $1 \mathrm{a}$ and $1 \mathrm{~b}$ ) and polystyrene (figure 1c) or natural cellular material as agglomerated cork (figure 1d). The structure is always multi-scale: beads are millimetric (from 2 to $8 \mathrm{~mm}$ ) whereas the size of the cells is a few tens of microns (figure 2). For the cellular materials studied in this paper, the size of the cells is relatively homogeneous for the polypropylene and polystyrene foams supplied by Knauf industries (the diameter is about 60 microns, figure $2 \mathrm{a}$ and $2 \mathrm{c}$ ). On the contrary, the structure of the PP foam given by JSP industries is heterogeneous (the diameter can reach 100 microns figure 2b). Concerning the cork, the cells are not spherical and can be instead considered as tubes of diameter 25 microns (figure $2 \mathrm{~d}$ ).

The mechanical behaviour of cellular material is well known. Under static or dynamic compressions, foam response shows three regimes: an elastic behaviour followed by a stress plateau corresponding to plastic yielding. Finally, for high strains, a rising hardening phase occurs due to foam densification. Many works treat of the effect of parameters such as foam density, strain rate or temperature $[2,3]$ but scarce are the studies which investigate the global response of the porous material taking into account the multi-scale structure of the foam, and the phenomena observed during a loading (local heterogeneity of the strain, buckling of bead and cell walls) [4]. Usually, elastic-plastic-rigid foam models are built from the homogenization of simplified cell (a cube, a polyhedron constituted of beams and walls) response [5]. The behaviour of this volume is identified from the beam and wall deformation and elastic-plastic-densification phases can be there modelled: the elastic phase corresponds to the compression of beams and 
walls, the plastic plateau is due to the buckling of beams and the stretching of walls and the densification appears when the cell volume tends towards zero. From this approach, the model can be finally identified measuring the average cell dimensions (diameter, wall thickness) and taking into account the mechanical properties of the constitutive material of the foam. The homogenisation of this microscopic model is the final step to obtain the macroscopic response of the cellular material. This numerical method is particularly appropriate if the foam is not multi-scale and if no deformation localisation is observed during a compression. For multi-scale foams, it is necessary to develop another approach in order to take into account the mesoscopic scale (beads) and the microscopic one (cells).

This article presents the first results of a project aiming to describe the behaviour of multi-scale foams under dynamic loading: firstly, dynamic compression tests were done to identify the macroscopic behaviour of these foams. Secondly, foam structure was analysed before, during and after impacts to observe physical phenomena caused by the loading at high strain rates. The experimental data obtained and the microscopic 3D observations acquired are necessary to develop a model for the material macroscopic behaviour taking into account bead and cell scales.

\section{Foam behaviour under dynamic loading}

\subsection{Macroscopic response of cellular material}

Four cellular materials have been studied: two polypropylene (PP), one polystyrene (PS) foam and agglomerate cork. For PP and PS foams provided by Knauf industry, four densities $\rho$ were tested: 70, 80, 90 and $100 \mathrm{~kg} / \mathrm{m}^{3}$. Densities of JSP polypropylene foams were 35,75 and $85 \mathrm{~kg} / \mathrm{m}^{3}$. Only one density of cork was tested: $\rho=270 \mathrm{~kg} / \mathrm{m}^{3}$. Several machines were used to qualify the behaviour of these cellular materials. A universal testing machine Zwick was used to identify the response of foams under quasi-static compression. For intermediate strain rates ( $\dot{\varepsilon}$ about $100 \mathrm{~s}^{-1}$ ), compression loadings were done on a drop tower. These tests permit to estimate the macroscopic response of foams even if the velocity of the projectile decreases during the impact. To complete the behaviour identification and to carry out dynamic compressions at constant strain rates, a new compression device was developed on a fly wheel [6] $\left(\dot{\varepsilon} \in[100,500] \mathrm{s}^{-1}\right)$. Lastly, Hopkinson bars were used to measure the foam response for higher strain rates $\left(\dot{\varepsilon}=1500 \mathrm{~s}^{-1}\right)$.

To qualify the foam macroscopic response on these devices, the stress $\sigma$ is calculated as the ratio of the measured force by the initial section $\mathrm{S}_{0}$ (Poisson's ratio is very low and the $\mathrm{S}$ section of the sample can be considered as constant). The true strain $\varepsilon$ can be calculated as an average deformation obtained by the Napierian logarithm of the ratio between the height $h(t)$ of the sample (function of time t) and its initial value. 


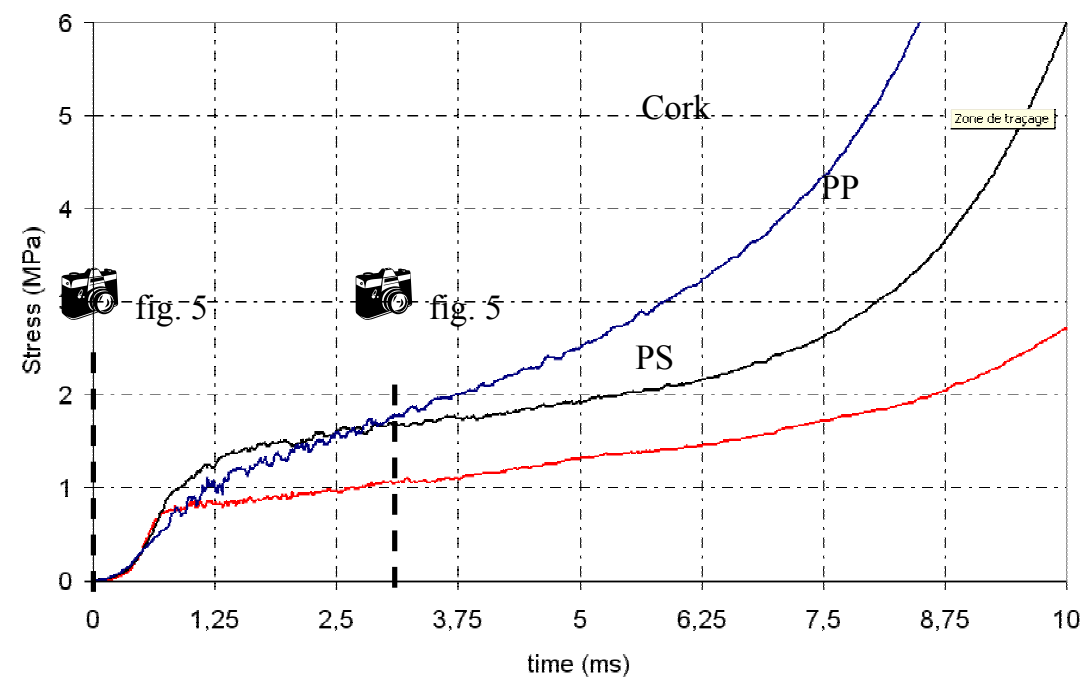

Figure 3: Stress vs. time response of polypropylene and polystyrene foams (density $95 \mathrm{~kg} / \mathrm{m}^{3}$ ) and cork for dynamic compressions $\left(\dot{\varepsilon}=100 \mathrm{~s}^{-1}\right)$.

Results showed figure 3 concern compressions at strain rates of $100 \mathrm{~s}^{-1}$ carried out on samples of PP, PS (from Knauf, same densities $\rho=95 \mathrm{~kg} / \mathrm{m}^{3}$ ) and agglomerate cork $\left(\rho=270 \mathrm{~kg} / \mathrm{m}^{3}\right)$. The evolutions of the stress as a function of time are similar for the three cellular materials. For these 3 tests, the compression duration is close to $10 \mathrm{~ms}$ and these stress evolutions correspond to the classical behaviour of foam: an elastic phase followed by a plastic plateau and lastly the densification. On dynamic apparatus, although it is really difficult to measure with accuracy the elastic modulus, these results allow to compare the elastic behaviour of these foams. The elastic responses of PS and PP foams (provided by the same manufacturers, same densities) seem similar. The Young modulus of PP and PS as dense materials being close, it's logical that the elastic responses of these foams are close too. In the same way, since the yield stress of dense PS is twice smaller than PP, the plastic plateau appears for lower stress for PS foam. The fact that the yield stress $\sigma_{\mathrm{pl}}$ is higher for polypropylene foams than for polystyrene foams have been verified for all the different densities. Similarly, the slope $\mathrm{E}_{\mathrm{pl}}$ of the stress plateau (also designed as the tangent modulus) is larger for PP foams than for PS foams.

Dynamic compressions using a fly wheel apparatus were also performed on these foams for different densities. For example, figure 4 presents the stress vs. strain responses of PP foams (Knauf) for 5 different densities (from 61 to 108 $\mathrm{kg} / \mathrm{m}^{3}$ ). Only the elastic phase and plastic plateau were plotted. The yield stress $\sigma_{\mathrm{pl}}$ is increasing with density. Indeed, a higher density involves a higher quantity of polypropylene, corresponding either to smaller cells in higher number or to 
thicker cell walls. In both cases the foam is more resistant. Finally, the density appears also to have an influence on $\mathrm{E}_{\mathrm{pl}}$, the slope of the stress plateau (also known as the tangent modulus). This modulus $\mathrm{E}_{\mathrm{pl}}$ is greater for higher densities.

In conclusion, these compression tests performed at different strain rates (from quasi static to high strain rates) on several multi-scale foams of different densities, and different morphologies allow to constitute a useful experimental database. For each cellular material, the classical stress - strain curve was acquired and the corresponding parameters identified.

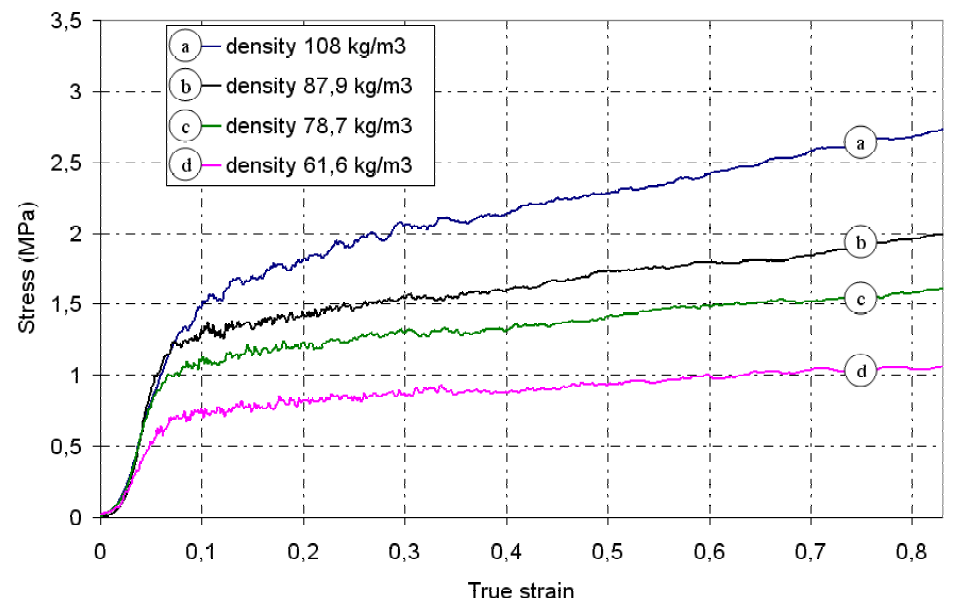

Figure 4: Stress vs. strain of polypropylene foams under dynamic compressions $\left(\dot{\varepsilon}=100 \mathrm{~s}^{-1}\right)$.

\subsection{Mesoscopic phenomena}

Dynamic compressions carried out in the fly wheel were recorded with a speed camera phantom V7 at 6688 frames per second (the time between two pictures is $150 \mu \mathrm{s}$ and the exposure time is $30 \mu \mathrm{s})$ with a resolution of $800 \times 600$ pixels. For each multi-scale foam studied, these short movies reveal the strong localisation of the deformation during plastic plateau. Figure 5 presents pictures extracted from these movies before loading $(\mathrm{t}=0 \mathrm{~ms})$ and during dynamic compression at $\mathrm{t}=3 \mathrm{~ms}$ for PP and PS foams (Knauf). On the first pictures (at $\mathrm{t}=0$ ) the bead structure of these foams is visible on the sample surface. The second pictures $(t=3 \mathrm{~ms})$ show the sample deformed during the plastic plateau (the curves presented figure 3 have shown that the stress plateau begins about $1.1 \mathrm{~ms}$ ). By comparison between the images at these two times, it is obvious that the deformation is strongly localised during the plastic plateau of these samples. One can see (particularly on PS, figure 5b) some foam beads which have been clearly damaged (darkened areas). This damage is diffused and located in the centre of the sample. The damaged layer is perpendicular to the compression axis and the beads located above and below this layer do not seem to be damaged. The same 
remarks can be done for PP foams (figure 5a); the foam damage appears also in the centre of the sample. The initial position of the damage layer seems however to be stochastic.

To complete this study, a micrographic analysis of compressed samples was carried out in order to confirm the heterogeneity of the foams damage. The SEM picture (figure 6) shows the residual deformation of PP foam after impact. At the mesoscopic scale, one can observe many bands of plastically deformed cells, with their fine walls buckled under loading. Other bands, yet intact, have kept their initial shapes. This damage mechanism, by local buckling of cell walls, explains the stress-strain curves; the plastic plateau corresponds to the propagation of foam cells buckling. At mesoscopic scale, bead walls have been also deformed by buckling. The damage mechanism can be thus considered multi-scale.

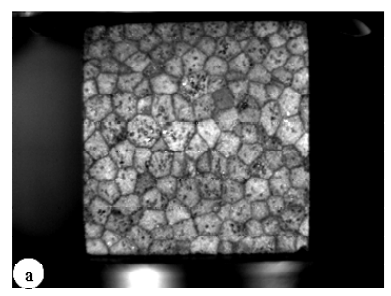

$\mathrm{t}=0 \mathrm{~ms}$

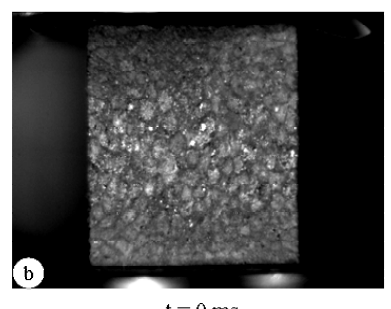

$\mathrm{t}=0 \mathrm{~ms}$

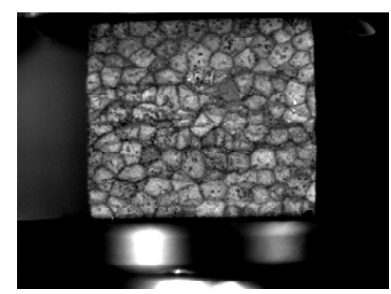

$\mathrm{t}=3 \mathrm{~ms}$

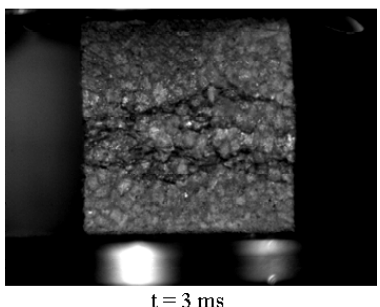

Figure 5: Pictures of foam samples $(\mathrm{a}-$ polypropylene, $\mathrm{b}$ - polystyrene) before and during dynamic compression (after $\mathrm{t}=3 \mathrm{~ms}$ ).

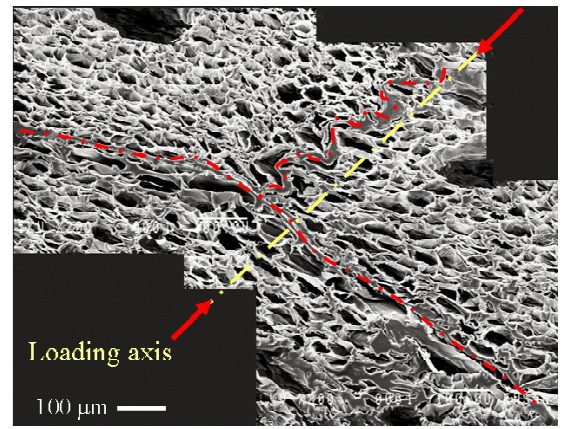

Figure 6: $\quad$ SEM view of PP foam sample after dynamic compression. 
The analysis of these pictures revealed the phenomena of strain localisation of these cellular materials during dynamic compression. The main ruin mechanism observed on these foams is the buckling of bead and cell walls. Lastly, if the damage appears systematically in layers perpendicular to the compression, its initial position cannot be predicted. As a first hypothesis, one can suppose that the variability of the bead density has an influence on the damage location.

A complementary investigation of the deformation mechanism has been initiated to validate (or reject) this assumption.

\subsection{Bead density effect on local deformation}

To complete this study, micro tomography technique was considered to observe the deformation inside the foam structure at different scales. The experimental method consists in carrying out several interrupted impact tests on a sample using a drop tower, and acquiring a micro tomograph in between each impact $[7,8]$. An image of the foam structure is taken before the first impact and after each dynamic compression; a device of dynamic compression was developed to control the maximum sample deformation during each impact and maintain the sample in compression during the micro tomographic measurement. The data presented in this paper have been obtained on the BM05 beam line at the European Synchrotron Radiation Facility (ESRF) in Grenoble (France). With this approach, first results of micro tomographic reconstructions reveal in $3 \mathrm{D}$ the state of the foam structure.

The behaviour described in this section is based on observations made in a vertical plane inside the 3D micro tomograph (sample of diameter $10 \mathrm{~mm}$ and height $10 \mathrm{~mm}$ ).

Figure 7 shows a vertical cross-section of the sample prior to impact. The pixels of high intensity correspond to points of dense material (within a bead wall for example). The reconstructed image is of good quality since one can observe the large air bubbles and closed cells are also distinguishable. At a larger scale, the geometry of the compacted beads can be identified as a classical polyhedral structure. This geometry can be followed for the successive impacts. In order to confirm the phenomena of strain localisation, one chooses to highlight the behaviour of 5 beads after each impact. A numerical filter was applied on this zone of the image (figure 7) to better distinguish bead structure. The same filter was applied on images reconstructed after each impact (figures $8 \mathrm{a}, \mathrm{b}$ and $\mathrm{c}$ ).

From these pictures, the state of deformation of each bead can be estimated and the analysis of the deformation of bead wall reveals that the loading can not be considered as an uni axial compression at the mesoscopic scale, the local loading seems more complex because the local deformation of bead walls corresponds certainly to a deformation resulting from a combination of compression and shear. The shape of bead 4 (figure 9) after each impact clearly confirms this hypothesis.

From this micro tomographic measurement, vertical strain $\varepsilon$ is also calculated (in the compression axis) and variation of density between beads can be estimated from the average of the grey level of each bead [8]. 


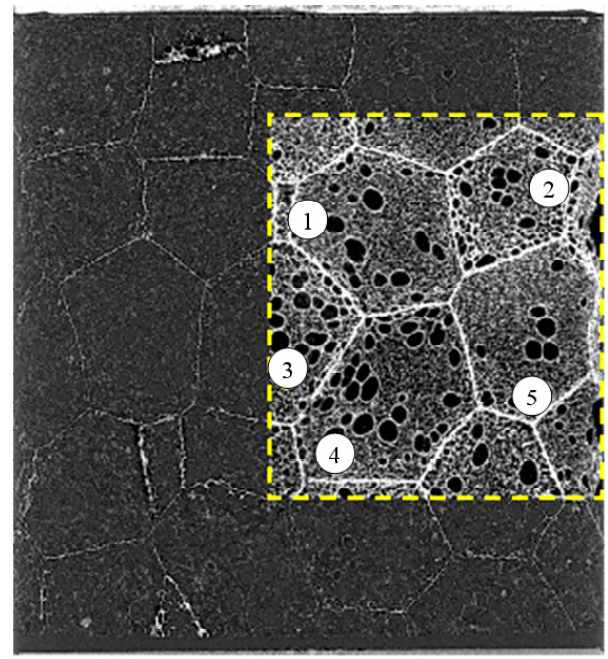

Figure 7: Vertical section (obtained by micro tomography) of polypropylene foam sample before impact.

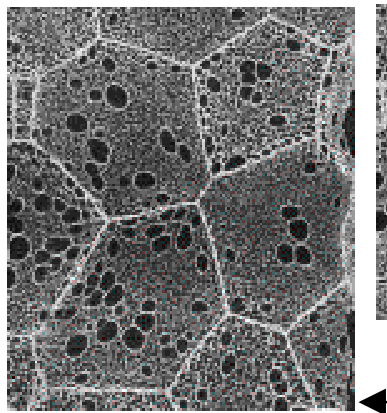

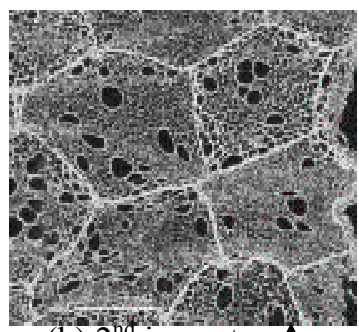

(b) $2^{\text {nit impact }}$

(a) $1^{\mathrm{er}}$ impact

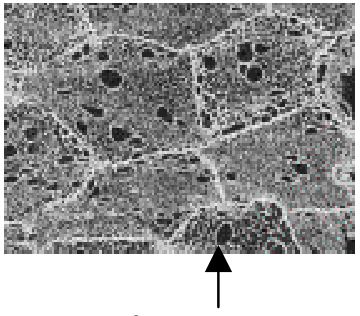

(c) $3^{\mathrm{e}}$ impact

Figure 8: Vertical sections (obtained by micro tomography) of the same polypropylene foam sample after impacts.

After the first impact, these 5 beads are slightly deformed. The strain value calculated is beyond $4 \%$ whereas the strain imposed on the sample is $10 \%$ (table 1). In fact, the strain analysis on the complete sample shows that deformation is principally localized on the upper and lower part of the sample, near the punches. For the second impact on the same sample, the macroscopic strain imposed is $30 \%$. Strong heterogeneous deformation of the 5 beads can be observed on Figure $8 \mathrm{~b}$. This can be verified on the strain value of each bead (table 1): The strain of bead 4 is four times bigger than the strain of bead 2 whereas the density (estimated from the average of grey level) of this last one is significantly higher. 
If we compare density of beads (or grey level) and their strain, a correlation between these two parameters cannot be established; higher density of a bead does not involve lower strain. On the contrary, in some case, beads of high density are more deformed. These observations can also be done for the third impact (strain imposed of $50 \%$ ). One can note a strong heterogeneity of bead strain and there is no direct relation between bead density and strain. Lastly, for the fourth impact (sample strain is $70 \%$ ), bead strain values are more homogeneous since the densification of the complete sample is reached.

To conclude, this last study performed using the micro tomographic technique has confirmed the strong localisation of bead deformation during dynamic compression within the material and not only on the sample external surface. The analysis of these last results has also revealed that a simple relation between bead density and deformation cannot be established. Phenomena observed are more complex and the localisation of the damage in cellular material depends certainly on a combination of several factors such as local density, obviously, but also defaults in the foam structure and morphology at both, meso and micro, scales.

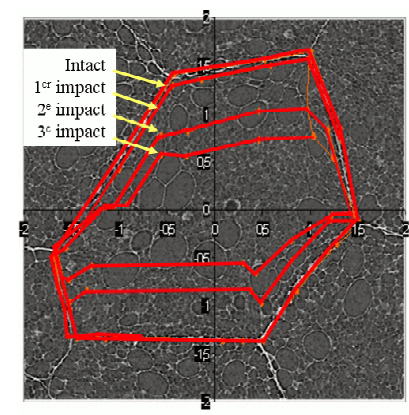

Figure 9: Sections of the same polypropylene bead $\left(\mathrm{N}^{\circ} 4\right)$ after several impacts.

Table 1: Strain values of beads marked on figure 7 and of the complete sample for the different interrupted impacts.

\begin{tabular}{|c|c|c|c|c|c|c|}
\cline { 3 - 7 } \multicolumn{1}{c}{} & \multicolumn{3}{c|}{ Strain (\%) } \\
\cline { 2 - 7 } \multicolumn{1}{c|}{} & $\begin{array}{c}\text { Surface } \\
\text { (pixel) }\end{array}$ & $\begin{array}{c}\text { Grey } \\
\text { level }\end{array}$ & Impact 1 & Impact 2 & Impact 3 & Impact 4 \\
\hline Bead 1 & 250522 & 77,7 & $-3,7$ & $-20,0$ & $-34,0$ & $-61,6$ \\
\hline Bead 2 & 153920 & 81,3 & $-1,7$ & $-8,6$ & $-24,2$ & $-54,5$ \\
\hline Bead 3 & 245959 & 77,6 & 0,0 & $-24,0$ & $-41,2$ & $-64,3$ \\
\hline Bead 4 & 274272 & 78,2 & $-4,0$ & $-41,4$ & $-59,2$ & $-72,9$ \\
\hline Bead 5 & 225409 & 81,2 & $-4,0$ & $-35,2$ & $-50,6$ & $-67,3$ \\
\hline Sample & $460.10^{4}$ & 82,2 & $-10,0$ & $-30,0$ & $-50,0$ & $-70,0$ \\
\hline
\end{tabular}




\section{Conclusion}

Dynamic compressions carried out on several multi-scale foams allowed to identify the global response of these cellular materials. The elastic step, the plastic plateau, during which the material is progressively damaged, and the final step, densification, have been characterized. The influence of density and strain rate on the foam behaviour was highlighted.

These macroscopic measurements were complemented by microscopic analysis performed using use of High-Tech apparatus (high speed camera, SEM, micro tomography). At this scale the foam damage appears severe on layers perpendicular to the loading direction and develop from this localization band. The heterogeneity of the strain in the sample is then significant. The deformation mechanism of fine wall cells and larger expensed grain walls is mainly buckling. This damage mechanism, by local buckling of cell and bead walls, explains the stress-strain curves; the plastic plateau corresponds to the buckling propagation on foam structure. This damage mechanism is multi-scale.

The initiation and propagation of buckling are complex and the localization of the damage in a cellular material structure depends certainly on a combination of local beads density and foam morphology at the meso and micro scales. One can suppose that it is the combined effect of the multi-scale structure and of the heterogeneous density field which creates a specific deformation field for a dynamic compression.

\section{References}

[1] Gibson L. and Ashby F.: "Cellular solids. Structures and properties", Edition: Cambridge Solid State Science Series.

[2] Chen W., Lu F., Winfree N.: "High-strain-rate compressive behavior of a rigid polyurethane foam with various densities", Experimental Mechanics, March 2002 vol. 42, no.1.

[3] Mills N.J., "Micromechanics of polymeric foams", Proceedings of the 3rd Nordic meeting on Materials and Mechanics, May 2000, Aalborg, Denmark, 45-76.

[4] Viot P. and Vacher P., "Identification of foam behavior under dynamic loading by the use of particle imaging techniques", Revue Matériaux et Techniques, hors série:39-43, December 2004. ISSN 0032-6895.

[5] Patel M.R and Finnie I., "Structural Features and Mechanical Properties of Rigid Cellular plastics", J. of Materials, Vol. 5, No. 4 pp 909 - 932

[6] Viot P., Beani F. and J-L. Lataillade, "Polymeric foam behavior under dynamic compressive loading”, J. Mater Sci, 2005, vol. 40, p. 5829-5837.

[7] Viot P. and Bernard D., "Impact test deformations of polypropylene foam samples followed by micro tomography", J. Mater Sci, vol 41 (2006) 1277.

[8] Viot P., Bernard D. and Plougonven E., "Phenomenological study of polymeric foam deformation under dynamic loading by the use of micro tomographic technique", Journal of Materials Science, (in press) 